\title{
THE FORMATION OF THE SHAMBLES AND OTHER BANKS BY TIDAL STIRRING OF THE SEAS
}

\author{
R. D. PINGREE* \\ Institute of Oceanographic Sciences, Wormley, Surrey
}

(Figs. 1-9)

\begin{abstract}
Tidal stirring of the seas in the neighbourhood of headlands, promontories and islands may concentrate sediments and result in the formation of tidal banks. At regular, smallscale promontories, symmetric bank development may occur. The situation at Portland Bill is examined in detail where bank development occurs preferentially in the anticlockwise eddy to the east, resulting in the formation of the 'Shambles', and some effects of the Earth's rotation are considered.
\end{abstract}

\section{INTRODUCTION}

It is well known that if a beaker of water, to which a little sand has been previously added, is stirred, then the sand moves along the bottom and accumulates in the centre. The result is the same whether the beaker is stirred clockwise or anticlockwise. It is perhaps less obvious, but equally true, that if the beaker is placed on a rotating table, then stirring clockwise and anticlockwise produces different effects. Any stirring rate in the sense of rotation may produce a concentration of sand towards the centre (better effects may be observed with less dense material - tea leaves for example). However, if the stirring is against the rate of rotation, then it is necessary to exceed the rotation rate before sand will accumulate in the centre of the beaker. In an analogous manner, 'spinup' of the table produces dispersion of bottom material, whereas spin-down results in a concentration of material towards the centre of the beaker.

These simple concepts have found little quantitative application to the geophysical problems of sandbank formation, on a rotating globe, by the tidal stirring of the seas. This is because reliable information on circulations in bays and off headlands has been difficult to obtain. However, a numerical model of the tides in the Portland region has derived the tidal circulations and the residual flows in the neighbourhood of Portland Bill, $\overline{\mathbf{u}}$, have been confirmed by direct observations (Fig. 1) (Pingree \& Maddock, $1977 a, b)$. The tidal eddies result from the different flood and ebb patterns of the tide due to the protruding shape of the submarine topography and coastline and are generated by the transfer of vorticity from the tide to the mean circulation (see Appendix I). In these eddies, the mean vorticity, $\omega$, is comparable with the vorticity, $f$, due to the Earth's rotation (Fig. 2) (see Appendix II). In the anticlockwise eddy, the Earth's rotation and the tidal stirring are in the same sense and conditions are suitable for sandbank development. In the clockwise eddy, tidal stirring and Earth's rotation are opposed. Thus nature

* Present address: The Laboratory, Marine Biological Association, Citadel Hill, Plymouth. 

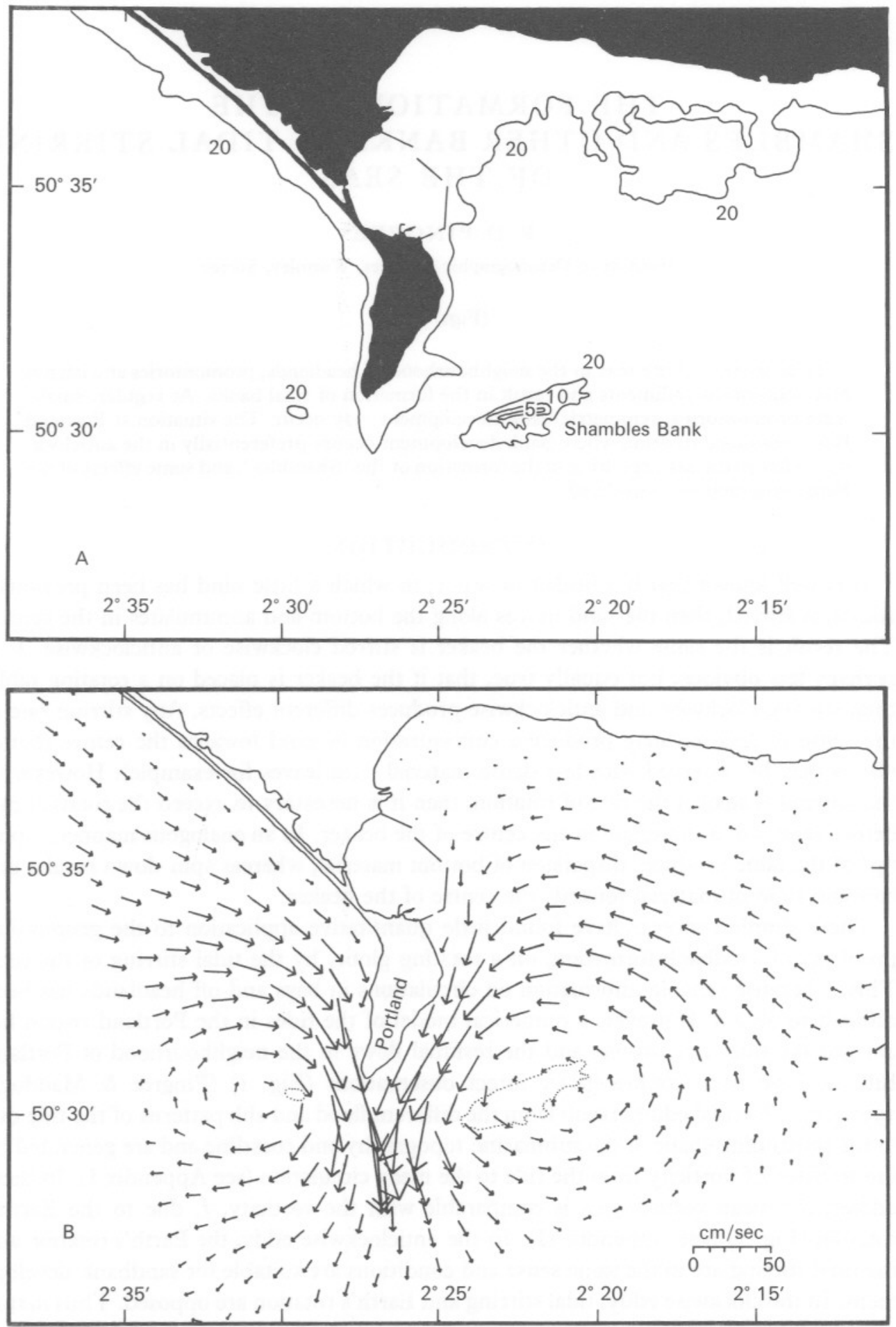

Fig. 1 A. Portland region. Water depth contours (m). B. Residual flows, $\overline{\mathbf{u}}$, in the neighbourhood of Portland Bill showing clockwise and anticlockwise eddies. Also shown are the positions of the Shambles Bank east of Portland Bill and a small bank to the west. (20 m contour.) 
has set up, on a 10-kilometre scale, our initial laboratory experiment with the beaker. In this paper, advantage is taken of this natural situation to examine to what extent these simple concepts can be applied to tidal bank formation. In particular, the situation at Portland is examined where tidal bank formation takes place preferentially in the anticlockwise eddy east of Portland Bill, resulting in the formation of the Shambles. It is shown later that, in addition to the accumulation of material by tidal stirring, tidal scouring is likely to play an important role in bank formation.

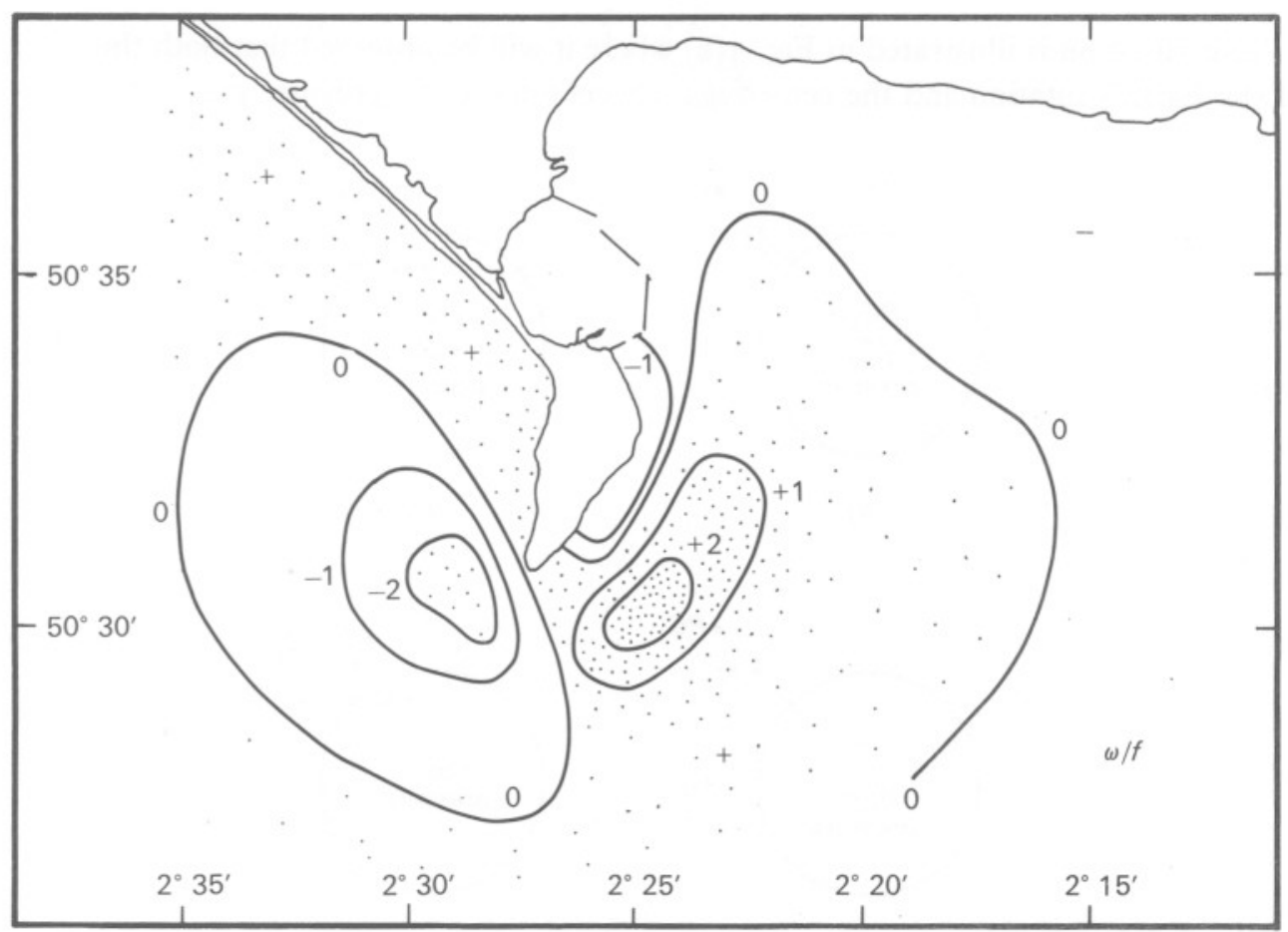

Fig. 2. Mean vorticity of the flow, $\bar{\omega}$, in contours of $\bar{\omega} / f$. Concentration is only likely to take place in the shaded regions (see text).

Many other promontories would appear to have tidal bank development fitting these simple concepts. For example, in the English Channel, the Skerries east of Start Point, the Goodwin Sands to the east of the South Foreland promontory and, in the Bristol Channel, Lundy Island, which has banks associated with all four eddies, and is considered further in Appendix II.

\section{METHOD AND RESULTS}

Balance of forces in anticlockwise and clockwise eddies

It is instructive to consider some general features of circular motion, even though these are not strictly applicable to the tidal situation. 


\section{Anticlockwise eddy}

Frictionless case. In the frictionless case, the forces to consider in an anticlockwise eddy are the centrifugal forces, $C F$ (arising from the curvature of the water flow), the Coriolis forces, $C$ (i.e. effect of the Earth's rotation) and the pressure gradient forces, $P G$ (that arise from the slope of the sea surface). In balance

$$
C F+C+P G=0 .
$$

This situation is illustrated in Fig. 3(a) where it will be observed that both the effects of the Earth's rotation and the centrifugal effects oppose the pressure gradient.

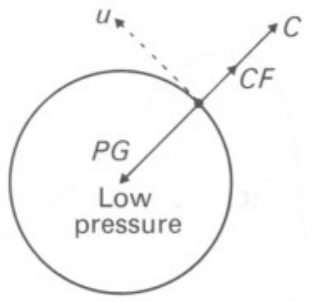

(a)

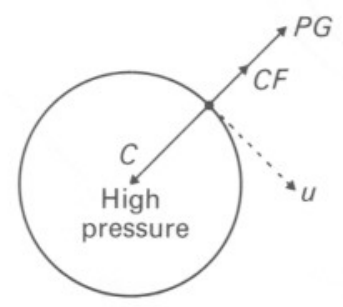

(c)

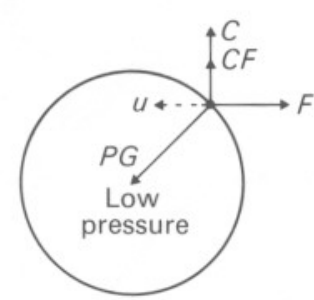

(b)

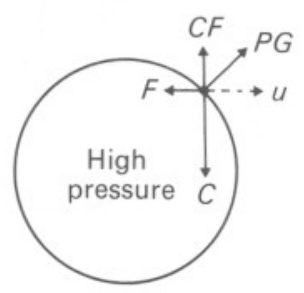

$(d)$

Fig. 3. Schematic representation of the balance of forces. (a) Anticlockwise flow with no friction where the Coriolis force, $C$, and centrifugal force, $C F$ balance the pressure gradient force, $P G$. (b) Modifying effect of bottom friction resulting in flow towards the low-pressure centre. (c) Clockwise flow with no friction where the pressure gradient force, $P G$, and the centrifugal force, $C F$, balance the Coriolis force, $C$. (d) Modifying effects of bottom friction resulting in flow away from the high-pressure centre.

Frictional effects. To demonstrate bottom convergence towards the centres of the eddies it is necessary to consider the effects of friction in modifying the vertical structure of the mean flow.

The full balance of forces must include a frictional force, $F$. In balance

$$
C F+C+P G+F=0
$$

Close to the bottom, the reduction of mean flow by bottom friction results in the reduc- 
tion of centrifugal and Coriolis forces, though the pressure gradient, which results from the sea surface slope, remains the same. In the limit, the balance now becomes

$$
P G+F=0,
$$

with the water near the bottom moving down the pressure gradient towards the lowpressure centre, resisted only by friction effects (Fig. $3 b$ ).

If the mean flow for the whole water column is non-divergent, and since it has been shown that the water close to the bottom is convergent, then upwelling must occur at the centre of these anticlockwise eddies followed by a divergence of water near the sea surface. Sediments moving close to the bottom will converge on the low-pressure centre, where material denser than sea water will accumulate.

\section{Clockwise eddy}

Although clockwise and anticlockwise eddies appear similar, an important difference exists. In the anticlockwise eddy, the Coriolis effects and the curvature effects act in the same direction and oppose the pressure gradient. In the clockwise eddy, the curvature effects and the Coriolis effects are opposed and therefore, for similar current strengths,

\section{Table 1. Symbols used in the text}

$\begin{array}{ll}t & \text { time coordinate } \\ x, y & \text { horizontal spatial coordinates } \\ E & \text { surface elevation } \\ h & \text { water depth } \\ \mathbf{u}=\overline{\mathbf{u}}+\mathbf{u}^{\prime} & \text { total horizontal velocity } \\ \nabla_{\wedge} \mathbf{u}=\omega=\bar{\omega}+\omega^{\prime} \text { total vorticity } \\ \mathbf{u}^{\prime} & \text { periodic tidal component of the flow } \\ a & \text { amplitude of the periodic tidal flow } \\ \mathbf{\mathbf { u }} & \text { residual flow with components } \bar{u}, \bar{v} \\ \omega^{\prime} & \text { periodic tidal component of vorticity } \\ \bar{\omega} & \text { mean vorticity } \\ \mathbf{W} & \text { wind velocity at a height of } 10 \mathrm{~m} \\ C_{D} & \text { drag coefficient }=0.0025 \\ C_{10} & \text { drag coefficient for wind at height } \\ C_{L} & \text { of } 10 \text { m } \\ \tau & \text { linearized drag coefficient } \\ & \text { bottom drag with component } \tau_{x}, \tau_{y}\end{array}$

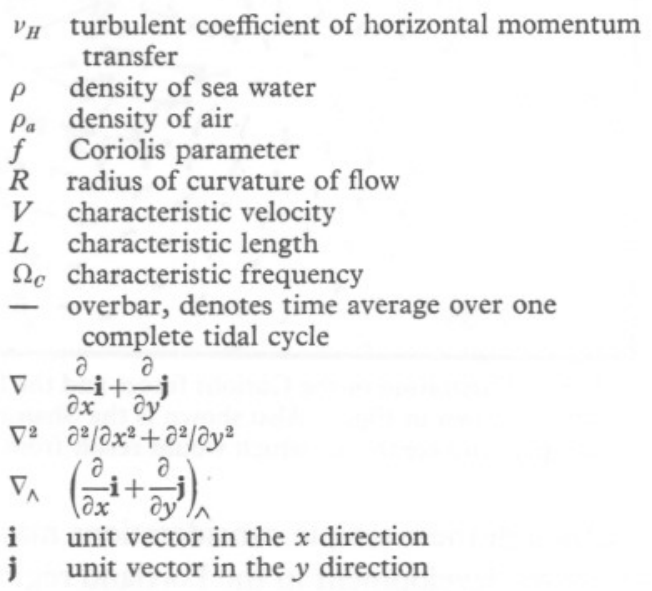

the pressure gradient term will be reduced. If the Coriolis term is larger than the curvature term, then the pressure gradient would be directed away from the clockwise eddy centre (Fig. 3c). The same reasoning as before would result in divergent flow near the bottom and surface convergence and downwelling at the eddy centre (Fig. 3 d). Floating material (in the absence of wind) would be expected to concentrate more readily in the clockwise eddy.

\section{Portland situation}

If the mean circulation in the Portland eddies (Fig. 1) was the only consideration in determining the mean forces at a position, then the Coriolis and inertial forces (arising from the velocity field accelerations) would appear as shown in Fig. 4. In accordance with the previous general considerations, the Coriolis forces and the forces resulting from the 
velocity field accelerations in the clockwise eddy to the west of Portland, are generally opposed, whilst in the anticlockwise eddy to the east of Portland the forces act in a similar sense. Since in this situation the Coriolis forces are comparable with the inertial forces, asymmetric bank development may be expected. If the residual flows and the corresponding balance of forces for these residuals were the only considerations, then the low-pressure region is centred over the Shambles Bank (Fig. 4).

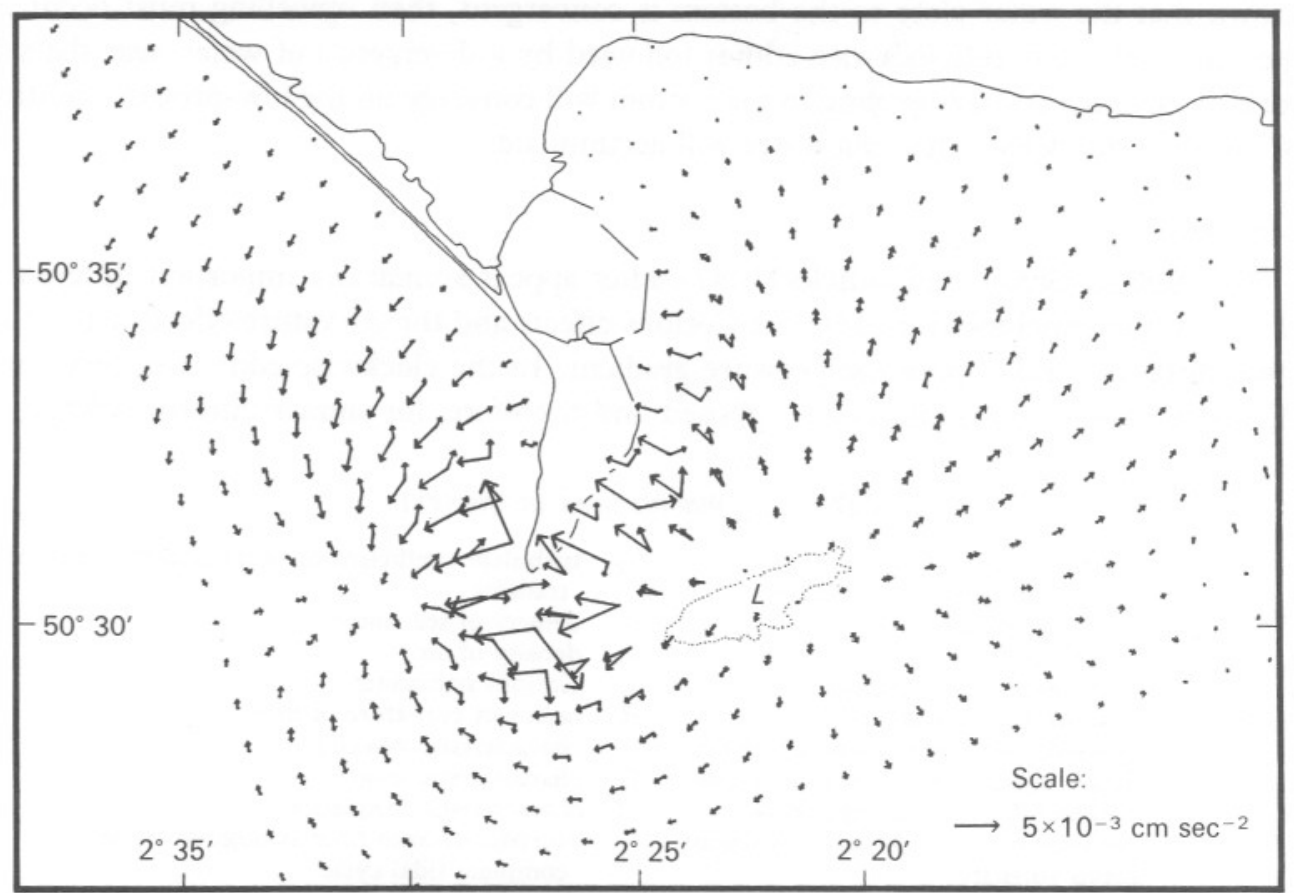

Fig. 4. Illustration of the Coriolis forces and the forces due to the field accelerations for the circulations shown in Fig. 1. Also shown is the Shambles Bank ( $20 \mathrm{~m}$ contour) and the position of the low-pressure centre, $L$, which would result from a balance of forces for the mean circulation.

Although these simple considerations may have considerable appeal in understanding tidal bank development in the Portland region, they overlook some important non-linear effects of tidal motion. Whilst it is true that the distribution of Coriolis forces for the mean circulation is also equal to the distribution of the mean Coriolis force experienced during the tidal cycle, this is not true for such non-linear terms as the advective term in the equations of motion.

\section{Mean sea level in the Portland region}

Non-linear effects completely modify this simple picture of the mean pressure distribution. When the tidal streams are running east, considerable curvature of the flow is experienced as the streams curve round Portland Bill. The normal accelerations will be balanced by a pressure gradient resulting in a reduction of pressure towards the tip of Portland Bill. When the tidal streams reverse, a similar effect occurs. Thus a net lowering 
of sea level occurs towards Portland Bill. In addition, the influence of headlands and promontories on the gross tidal flow leads to increased tidal streaming off headlands and weaker tidal velocities in adjacent bays (see Appendix I). Since the pressure drop due to this effect is also proportional to the velocity squared, a lowering of mean sea level following the flow must be expected in the neighbourhood of headlands (Fig. 5).

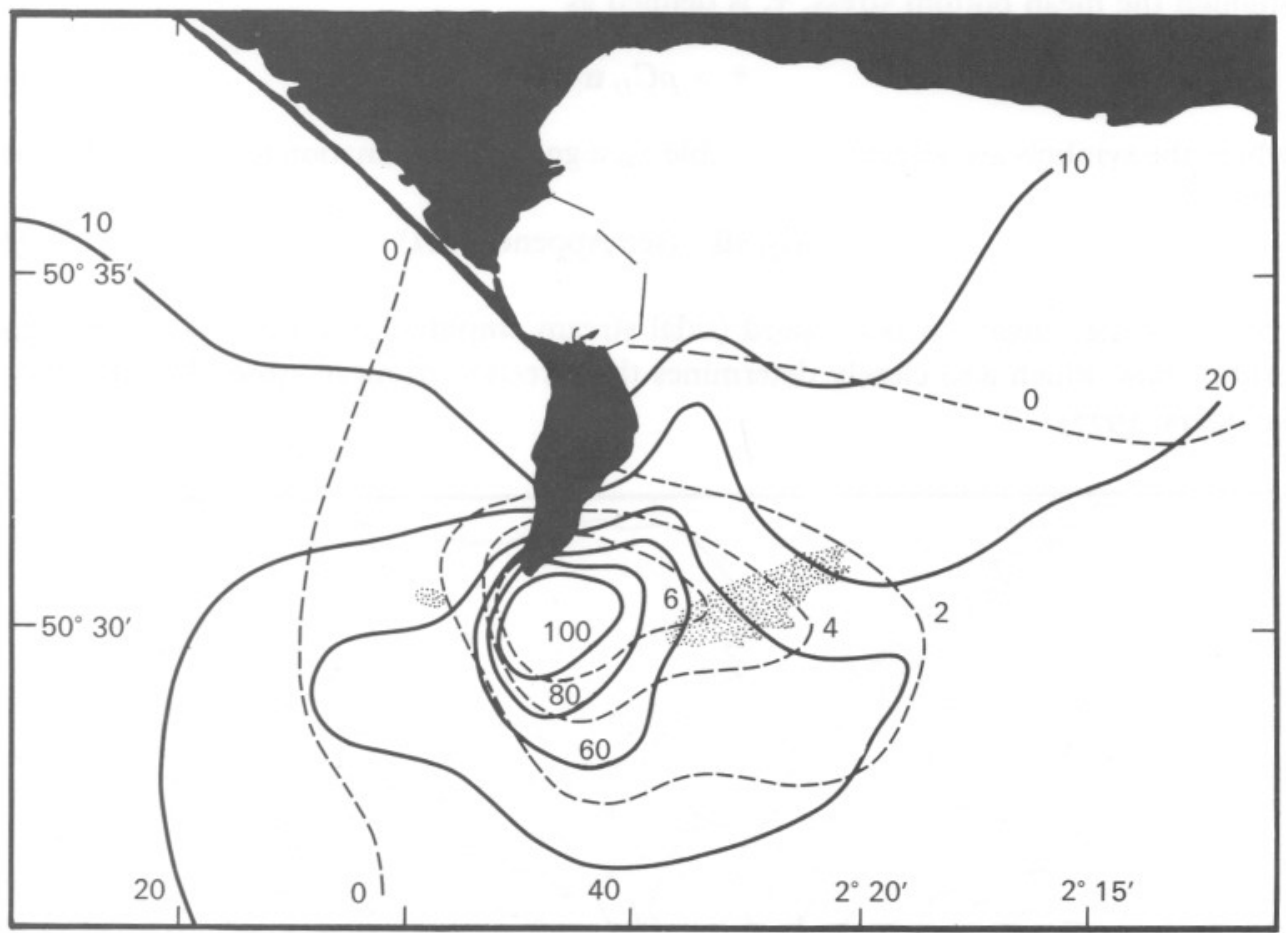

Fig. 5. The mean surface depression (in cm dashed line) including non-linear effects of tidal motion. Also shown is the distribution of maximum stress (dynes $\mathrm{cm}^{-2}$ ), which includes contributions from the $\mathrm{M}_{4}$ tide and residual flows.

\section{Maximum stress and mean stress over a tidal cycle}

It is important to realize that these latter effects on the pressure distribution will be most marked during maximum tidal streaming. Since tidal scouring may also be expected to be proportional to the velocity squared, the maximum stress $\tau_{\max }$, where

$$
\tau_{\max }=\rho C_{D} \times(\text { maximum speed })^{2},
$$

will also be greatest during this period of the tidal cycle (Fig. 5). It is argued that the high values of bottom stress towards the tip of the Bill will prevent the accumulation of material in the low-pressure region at the tip of the'Bill. However, it must still be expected that upwelling will occur in the low pressure centre, particularly during maximum anticlockwise streaming (see $\mathrm{R}_{0}$, Appendix II). Eroded material will be tranisported and deposited in the eddy which still persists when slack tide occurs at the tip of the Bill. 
The continual effects of scouring and deposition will result in a grading of material according to the distribution of maximum stress (Shields, 1936). Once the tidal bank has formed with the appropriately graded material, the tidal streams may continue to work the bank material. The direction of sandwave movement might be expected to follow the direction of the stress averaged over a tidal cycle (Fig. 6). It is clear that these directions closely follow the directions of the residual flows (Fig. 1b). This results from the fact that although the mean bottom stress, $\bar{\tau}$, is defined as

$$
\bar{\tau}=\rho C_{D} \overline{\mathbf{u} \mid \mathbf{u}} \mid
$$

(where the symbols are as defined in Table 1), a good approximation to the mean bottom stress is

$$
\bar{\tau} \sim \rho C_{D} a \overline{\mathbf{u}} \quad \text { (see Appendix III) }
$$

where $a$ is the maximum tidal speed (tidal stream amplitude) at a point, and $\overline{\mathbf{u}}$ is the residual flow which also closely determines the direction of mean stress (Hunter, 1975; Saunders, 1977).

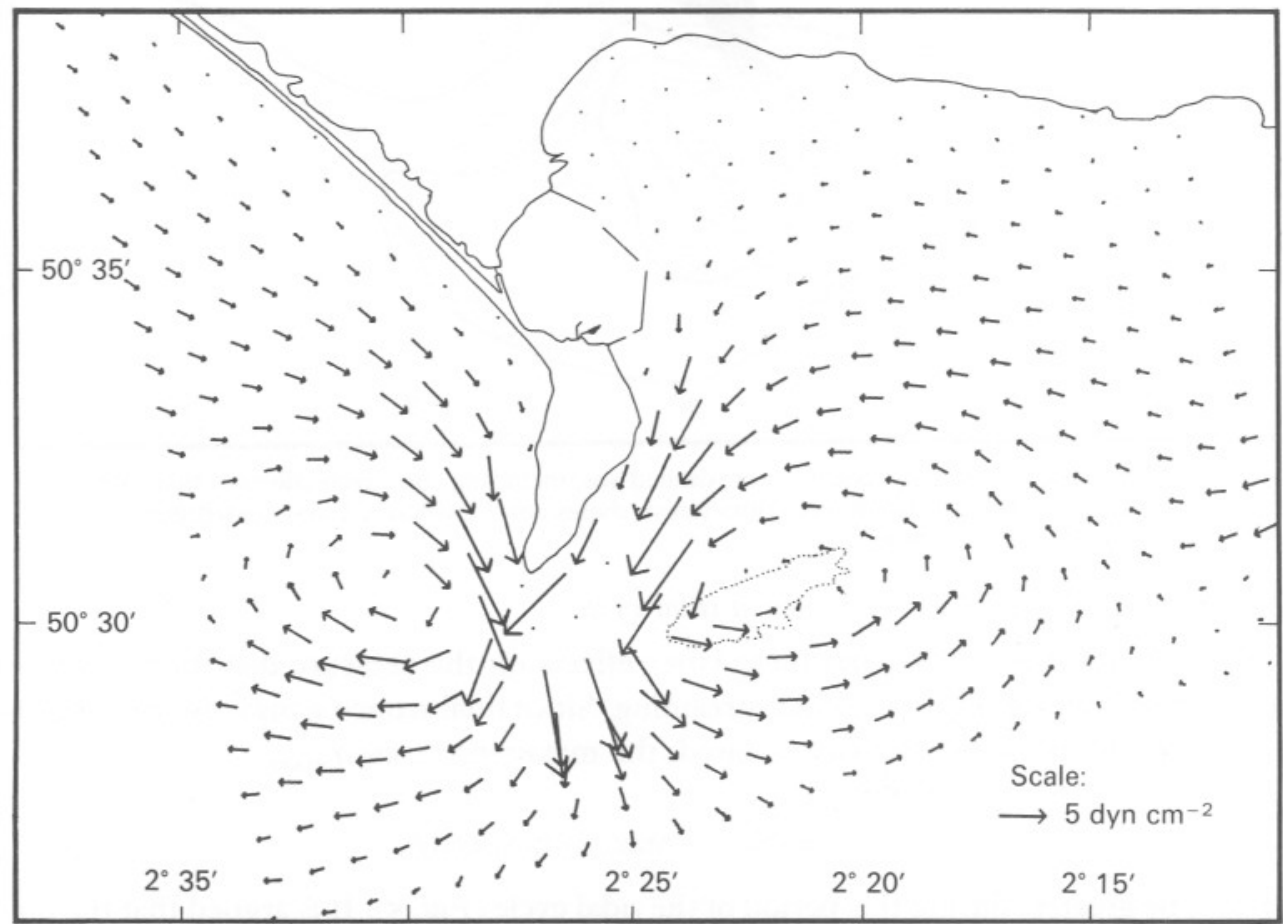

Fig. 6. Mean bottom stress over a tidal cycle. (For clarity some large values near the tip of Portland Bill have been omitted.) 


\section{Effects of weather}

Just as the springs to neaps cycle of tidal streaming must be expected to be important in erosion and deposition processes so too must the effects of weather be taken into account. Whereas the effects of swell may be important in determining threshold values for the movement of sediment, the wind stress acting on the sea surface will affect the residual flow and hence the balance of forces together with the distribution of mean bottom stress.

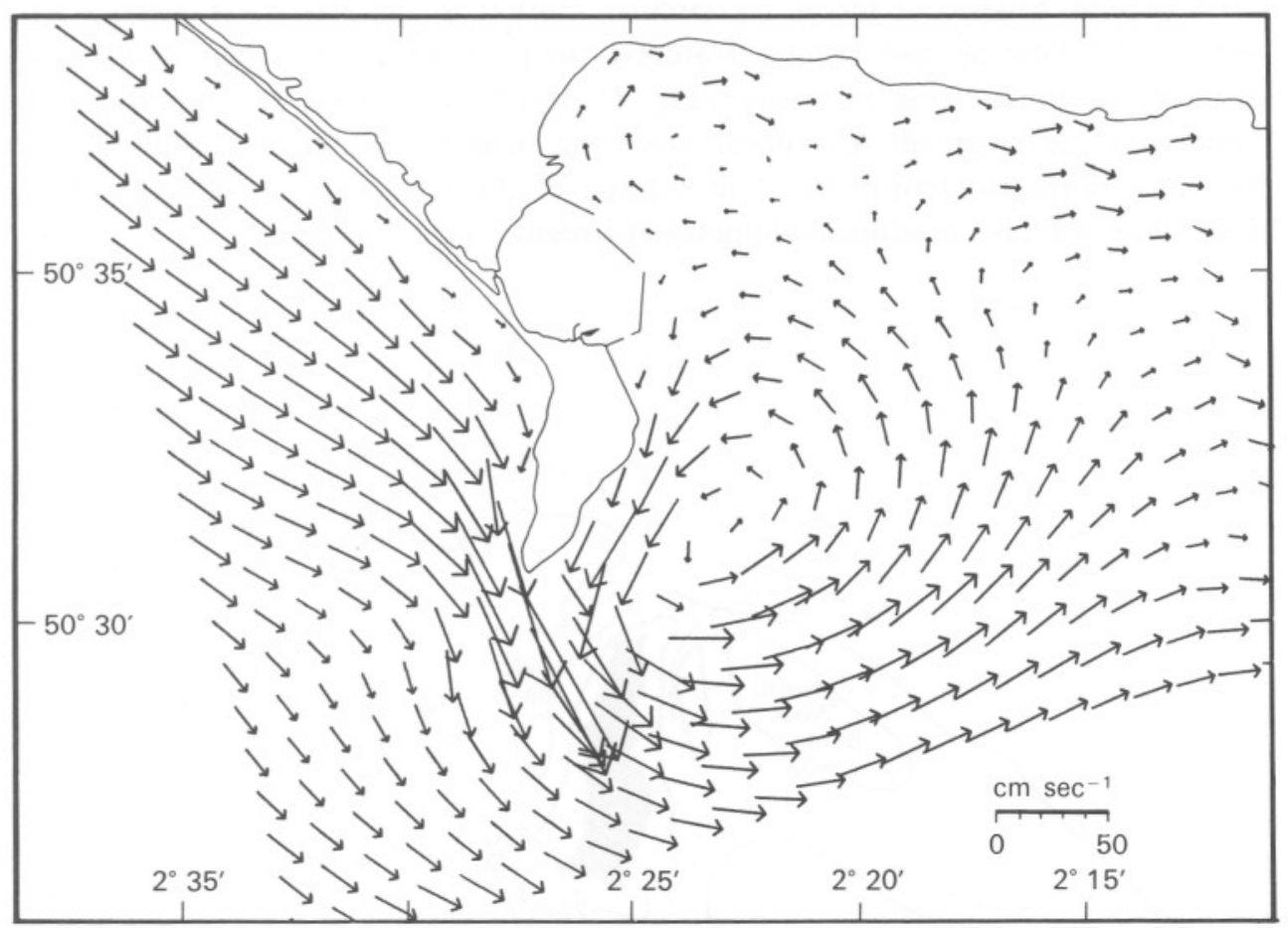

Fig. 7. Residual flow under gale force conditions. Wind WSW 40 knots.

No attempt will be made here to describe results of different wind strengths from different directions, but simply to summarize some salient features. Varying winds were blown over the region and the wind was assumed to be in geostrophic balance with the surface pressure. The wind stress is defined as

$$
\tau_{\mathbf{w}}=\rho_{a} C_{10} \mathbf{W}|\mathbf{W}|
$$

where $\rho_{a}$ is the density of air $\left(\sim 1.2 \times 10^{-3} \mathrm{~g} \mathrm{~cm}^{-3}\right), C_{10}$ is the drag coefficient $\left(\sim 1.3 \times 10^{-3}\right.$, Phillips, 1966) and $\mathbf{W}$ is the wind velocity at a height of $10 \mathrm{~m}$.

For winds less than about 20 knots there is little effect on the Portland eddies since

$$
\text { mean wind stress, } \tau_{\mathrm{w}}<\text { mean bottom tidal stress, } \bar{\tau} \text {. }
$$

For much increased wind speeds this is no longer true, and under gale conditions 
considerable modification can be expected. For a 40 knot WSW gale (i.e. blowing along the axis of the English Channel) the effect of the wind is to eliminate completely the clockwise eddy to the west of Portland Bill (Fig. 7). Such conditions are likely to destroy any tendency for bank formation west of Portland and increase transport of material around the Shambles bank east of Portland. Bank structures may be determined by relatively few natural catastrophic events (see for example Hails, 1975).

\section{DISCUSSION}

The general balance of forces for circular motion is considered. If inertial forces dominate the flow around regular features, then clockwise or anticlockwise eddies will result in similar bank formation (Fig. 8). If Coriolis effects are important, then asymmetrical development may occur favouring bank formation in the anticlockwise eddy in the northern hemisphere. The shaping of the submarine topography close to the coastline will also produce asymmetry at irregular promontories.

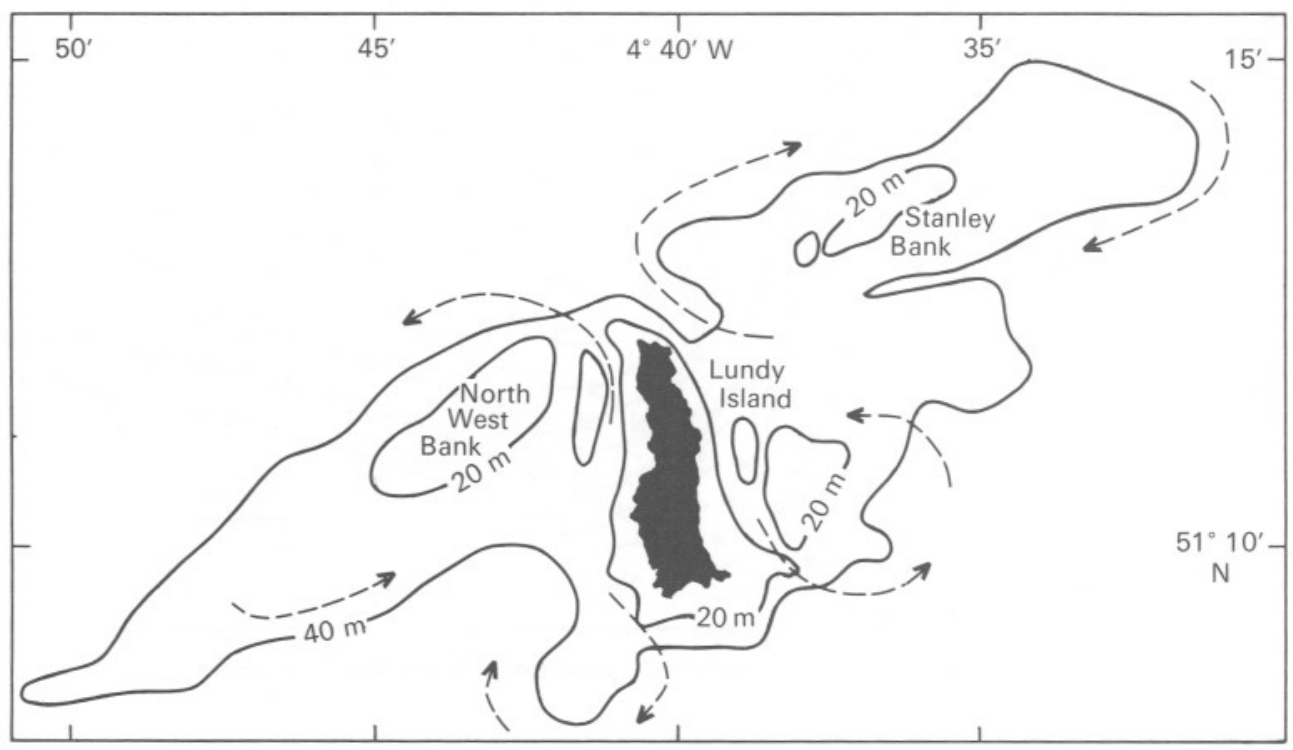

Fig. 8. Bottom contours in the neighbourhood of Lundy Island showing submarine development (depth, m). Arrows indicate the residual circulations.

Headlands are shown to produce characteristic eddy patterns of tidal flow which are the result of vorticity transfer from the tide to the residual flow (Fig. 9, see Appendix I). As a particular case, the residual tidal circulation patterns that develop east and west of Portland are considered. It is observed that the Shambles bank lies in the centre of the anticlockwise eddy to the east of Portland and only vestigial bank development occurs in the clockwise eddy to the west. Although instructive, the simple balance of forces for circular motions cannot be applied directly to this situation. To fully account for bank development at Portland it is necessary to suppose that the high bottom stress region that occurs during maximum tidal streaming produces scouring which prevents the 
accumulation of sediment at the tip of Portland Bill, thereby preventing any tendency for spit formation which may sometimes develop.

The overall picture of tidal bank formation presented is a dynamic one representing more than a passive settling out of material in regions of low tidal energy only. Bottom material is either lifted into suspension or dragged along the bottom around the eddy

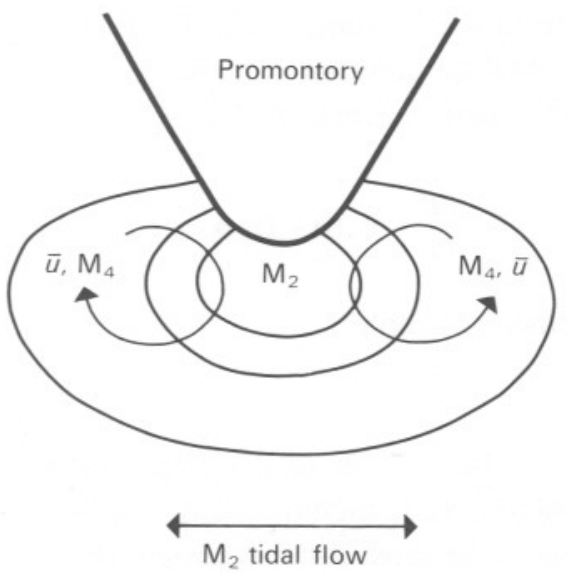

Fig. 9. Schematic representation of the flow in the neighbourhood of a promontory. The contours represent $\mathrm{M}_{2}$ tidal vorticity during maximum $\mathrm{M}_{2}$ tidal streaming and increase towards the promontory. The advection of $\mathrm{M}_{2}$ tidal vorticity by the $\mathrm{M}_{2}$ tidal flow generates $\mathrm{M}_{4}$ tidal vorticity and a residual circulation either side of the promontory, see equation $12 . \mathrm{M}_{2}$ tidal vorticity is generated by the term $-\nabla_{\wedge} \tau / h$, see equation 13 .

during conditions of high tidal stress. Once the bank forms an equilibrium profile, the material will still be further worked around the fully developed bank. Thus, if material were removed from the crest of the bank, it is expected that peripheral material would spiral in to take its place. This may take place gradually with each tidal cycle or suddenly under storm conditions. It must then be expected that dredging operations may result in erosion of material elsewhere.

I wish to thank Dr Linda Maddock for computing assistance and advice.

\section{APPENDIX I \\ Vorticity balance}

The vertically integrated equations of motion

The basic equations used in the development of the numerical model express the conservation of horizontal momentum and the conservation of mass. For numerical convenience these equations are vertically integrated to give equations in terms of the mean horizontal velocity, $\mathbf{u}$, of the total water column, $h+E$, where $h$ is the water depth and $E$ is the surface elevation. Assuming the water is of constant density and applying the hydrostatic approximation, the momentum equation can be vertically integrated to give

$$
\frac{\partial \mathbf{u}}{\partial t}+\mathbf{u} \cdot \nabla \mathbf{u}+2 \Omega \wedge \mathbf{u}=-g \nabla E-\frac{C_{D}}{h+E} \mathbf{u}|\mathbf{u}|+\nu_{H} \nabla^{2} \mathbf{u}
$$


where the time variations of the horizontal velocity vector, $\partial \mathbf{u} / \partial t$, are the result of accelerations arising from

(i) the advection of momentum, $\mathbf{u} . \nabla \mathbf{u}$, which is a good approximation for the advection term if vertical shears are small;

(ii) the effect of the Earth's rotation, $2 \Omega \wedge \mathbf{u}$;

(iii) the slope of the sea surface, $-g \nabla E$;

(iv) the frictional drag from the bottom, $-C_{D} /(h+E) \mathbf{u}|\mathbf{u}|$;

(v) the transfer of horizontal momentum by mixing, $v_{H} \nabla^{2} \mathbf{u}$.

The vertically integrated equation expressing the conservation of mass can be written as

$$
\frac{\partial E}{\partial t}+\nabla \cdot(h+E) \mathbf{u}=0,
$$

where the symbols are defined in Table 1.

\section{Potential vorticity}

If the frictional forces and the transfer of horizontal momentum by mixing are neglected, the sea surface elevation, $E$, is included with the water depth, $h$, and the slope of the sea surface is eliminated by cross differentiation, then, with use of the continuity equation (10) it is possible to derive the equation for the potential vorticity, $(f+\omega) / h$, of the water column, namely

$$
\frac{\partial[(f+\omega) / h]}{\partial t}+\mathbf{u} \cdot \nabla[(f+\omega) / h]=0 .
$$

This equation states that the potential vorticity of a water column cannot change as it moves from one place to another. This explains why tidal streams are weak in bays and strong off headlands. If the tidal streams are confined to follow coastal topography, the curvature of the flow in bays will be in opposite sense to the curvature off promontories. If the Coriolis parameter, $f$, is neglected and the relative vorticity, $\omega$, is everywhere zero then to maintain irrotational flow it is necessary that the tidal streams increase off headlands and decrease in small ( $\ll$ tidal wavelength) bays.

\section{Transfer of vorticity from the tide to the mean flow}

Including frictional effects and mixing but, for simplicity of argument neglecting effects due to the Earth's rotation, the vorticity equation can be written as

$$
\frac{\partial \boldsymbol{\omega}}{\partial t}+\nabla \cdot(\mathbf{u} \boldsymbol{\omega})=-\nabla \wedge \boldsymbol{\tau} / h+\nu_{H} \nabla^{2} \boldsymbol{\omega} .
$$

Now separating the velocity, $\mathbf{u}$, and vorticity, $\boldsymbol{\omega}$, into mean components $\overline{\mathbf{u}}, \overline{\boldsymbol{\omega}}$ and tidal component $\mathbf{u}^{\prime}, \boldsymbol{\omega}^{\prime}$ and remembering

$$
\begin{gathered}
-\nabla_{\wedge} \tau / h=\text { dissipation }+ \text { generation of vorticity } \\
\text { or }-\nabla_{\wedge} \tau / h=-C_{D} \frac{|\mathbf{u}| \boldsymbol{\omega}}{h}+C_{D} \mathbf{u}_{\Lambda} \nabla\left(\frac{|\mathbf{u}|}{h}\right)
\end{gathered}
$$


and averaging over a tidal cycle, we obtain

$$
\nabla \cdot\left(\overline{\mathbf{u}^{\prime} \boldsymbol{\omega}^{\prime}}\right)+\overline{\mathbf{u}} h \cdot \nabla(\overline{\overline{\boldsymbol{\omega}}})=-\frac{C_{L}}{h} \overline{\boldsymbol{\omega}}+\nu_{H} \nabla^{2} \overline{\boldsymbol{\omega}}
$$

where, for convenience, the frictional term (equation 13) has been linearized using the linearized drag coefficient, $C_{L}$ (see Appendix III).

The first term on the left-hand side of equation (14) represents the vorticity transfer from the tidal periodic current to the residual flow, and the first term on the right-hand side is the dissipation vorticity due to bottom friction. The second term on the left-hand side describes the redistribution of the mean vorticity by the mean flow itself and the second term on the right-hand side expresses the redistribution of mean vorticity by horizontal turbulence.

$\mathrm{M}_{2}$ tidal vorticity is derived as the tide curves around the Portland peninsula due to spatial changes in water depth and tidal stream amplitude as described by the second term on the right hand side of equation 13. Irrotational flow cannot be maintained at the headland and consequently $\mathrm{M}_{2}$ vorticity is generated at the headland with the same rotational sense as the curvature of the flow around the headland. Clockwise $\mathrm{M}_{2}$ vorticity is advected to the west when the tidal stream is to the west of Portland Bill, and anticlockwise $M_{2}$ vorticity is advected to the east when the general tidal streams run in an easterly direction. In fact, the $\nabla\left(\mathbf{u}^{\prime} \boldsymbol{\omega}^{\prime}\right)$ term generates clockwise vorticity west of Portland Bill and anticlockwise vorticity east of Portland Bill whether the $\mathrm{M}_{2}$ tidal streams are running strongly east or west. This process generates an $\mathrm{M}_{4}$ vorticity source, and a mean vorticity source or residual circulation (Fig. 9). These circulations exist even if no tidal bank develops and it is not correct to suppose that the tidal banks create the eddies, though the banks may ultimately modify the flow. Residual flows similar to those shown in Fig. $1(b)$ are obtained if the banks are removed.

The same arguments would lead us to expect anticlockwise vorticity around the Hammond Knoll and Haisborough Sand in response to $\mathrm{M}_{2}$ tidal vorticity generated as the tide follows the East Anglian coastline. Evidence for $\mathrm{M}_{2}$ tidal vorticity is gleaned from the fact that the tidal stream amplitude reaches maximum values $\left(\sim 3 \frac{1}{2}\right.$ knots $)$ some 6 nautical miles off the coast near Great Yarmouth where the tidal streams are only about 2 knots. However, inferred migration directions of some Norfolk sandbanks in this region (Caston \& Stride, 1970) have suggested that the circulations around the Norfolk Banks are on the contrary clockwise. Low values of $\mathrm{R}_{0} \sim 0.2$ (see Appendix II) for the maximum tidal flow along the East Anglian coast are consistent with gross asymmetry.

\section{APPENDIX II}

\section{Scale analysis}

In this Appendix the situation at Portland is compared with that at Lundy.

The relative importance of inertial effects and Coriolis effects can be defined conveniently from the Rossby number. The Rossby number, $R_{0}$, of the flow is the ratio of the inertial force to the Coriolis force

$$
R_{0}=\frac{\mathbf{u}^{2} / R}{f \mathbf{u}}=\frac{\mathbf{u}}{f R}
$$


For higher Rossby numbers, $R_{0} \gg 1$, stirring clockwise or anticlockwise produces convergence of water near the bottom resulting in conditions suitable for bank formation. For low Rossby number, $R_{0} \ll 1$, Coriolis forces dominate and for anticlockwise rotation (in the northern hemisphere) the flow near the bottom will be convergent, and directed towards the low-pressure centre. For clockwise rotation, with $R_{0} \ll 1$, the centre will be a high-pressure region and the flow near the bottom will be divergent and directed away from the centre. For $R_{0}=1$ the anticlockwise flow will have a central low pressure with convergent bottom flow but the clockwise flow will have no pressure gradient force and no tendency towards bank formation since the centrifugal force will just balance the Coriolis force; such motion is called 'inertial motion'.

The generation and dissipation of vorticity results in eddies on either side of headlands that may favour sandbank development. In some large-scale situations the effects of the Earth's rotation may be important. The distribution of mean vorticity in the Portland eddies is illustrated in Fig. 2, where the values of mean vorticity have been divided by the vorticity due to the Earth's rotation to provide contours of $\bar{\omega} / f$.

In the very simple case of solid rotation of the eddies, positive values of $\bar{\omega} / f$ may provide conditions suitable for the development of banks. For negative values, $\bar{\omega} / f$ must be less than -2 before bank formation may occur. Thus in this artificial situation bank development will not occur for $\bar{\omega} / f$, such that,

$$
-2 \leqslant \bar{\omega} / f \leqslant 0 .
$$

Hence we may anticipate preferential tidal bank development to the east of Portland Bill where positive values of $\bar{\omega} / f$ occur, and only marginal bank development in the west, where values more negative than -2 cover only a small geographical region.

For promontories or elongated islands, situated at right angles to the gross offshore tidal flow, it is convenient to define an approximate frequency scale, $\Omega_{C}$,

$$
\text { where } \Omega_{C}=\frac{1}{C_{D}} \frac{h}{l} \frac{V}{L}
$$

that may be used to compare bank development where $l$ and $L$ represent length scales associated with changes in depth $h$ and tidal stream amplitude $V$ in the vicinity of the headland. For simplicity we take $L$ as the length of the headland, which we also take as the characteristic length, $l$. For elongated islands, $L$ will be half the length of the island since eddies may develop at both ends.

As an example, let us consider tidal bank development at Portland and the Island of Lundy, where the water depth is $\sim 40 \mathrm{~m}$. Then for the Portland Headland $V \sim 150$ $\mathrm{cm} \mathrm{sec}^{-1}, L \sim 10^{6} \mathrm{~cm}$ and using $C_{D}=0.0025$ then

$$
\mathrm{R}_{0} \sim 1 \text { and } \Omega_{C} \text { Portland } / f \sim 2
$$

for Lundy Island $V \sim 150 \mathrm{~cm} \mathrm{sec}^{-1}$ and $L \sim 1 \frac{1}{2}$ nautical miles $\sim 3 \times 10^{5} \mathrm{~cm}$

$$
\mathrm{R}_{0} \sim 5 \text { and } \Omega_{C} \text { Lundy } / f \sim 27 .
$$

Consequently, tidal bank development at Lundy would be expected to be more symmetrically developed than at Portland, as is indeed observed (Fig. 8). As in the case of Port- 
land, the line of centres joining the North West Bank and the Stanley Bank is in the approximate direction of the orientation of the major axis of the tidal ellipse.

Other factors that effect the eddy development result from the shaping of the submarine topography and either side of the headland, and the angle the headland makes with the major axis of the tidal streams. The reduced banks lying to the south of Lundy reflect the relatively more rounded southern end of the Island of Lundy in comparison with the sharpness of its northernmost promontory.

\section{APPENDIX III}

\section{Analytical approximation to the mean bottom stress}

Although the mean bottom stress values have been derived from the numerical model (Fig. 6) for some situations they can be conveniently derived analytically (Hunter, 1975). For the Portland situation, where the tidal flow across the eddies is approximately rectilinear (ratio of major to minor axis $\sim>5$ ) and the tidal stream amplitude $a$ is much larger than the residual flow, $\overline{\mathbf{u}}$ then the mean bottom stress can be written as

$$
\left[\begin{array}{l}
\bar{\tau}_{x} \\
\bar{\tau}_{y}
\end{array}\right]=\frac{2 \rho a C_{D}}{\pi}\left[\begin{array}{ll}
2 & 0 \\
0 & 1
\end{array}\right]\left[\begin{array}{l}
\bar{u} \\
\bar{v}
\end{array}\right],
$$

where

$$
\bar{\tau}=\bar{\tau}_{x} \mathbf{i}+\bar{\tau}_{y} \mathbf{j}
$$

and

$$
\overline{\mathbf{u}}=\bar{u} \mathbf{i}+v \mathbf{j}
$$

(see Table 1 for definition of symbols and where $a$ is assumed along the $x$ axis).

Hence if the mean current $\overline{\mathbf{u}}$ is in the same direction as the tidal current then

$$
\bar{\tau}=\frac{4}{\pi} \rho C_{D} a \overline{\mathbf{u}} .
$$

If the mean current and the tidal current are at right angles then:

$$
\bar{\tau}=\frac{2}{\pi} \rho C_{D} a \overline{\mathbf{u}} .
$$

In both cases the mean bottom stress is in the direction of the residual flow, $\overline{\mathbf{u}}$. For other directions of residual flow and tidal stream orientation the difference in direction between the residual flow and the mean bottom stress can be determined from (18). The maximum difference is only $19.5^{\circ}$, which occurs when the tidal streams intersect the residual flows at $55^{\circ}$, and the mean bottom stress is then

$$
|\bar{\tau}|=\frac{2 \cdot 8}{\pi} \rho C_{D} a|\overline{\mathbf{u}}|
$$

\section{REFERENCES}

Caston, V. N. D. \& Stride, A. M., 1970. Tidal sand movement between some linear sand banks in the North Sea off northeast Norfolk. Marine Geology, 9, $\mathrm{M}_{3} 8$.

HaILS, J. R., 1975. Sediment distribution and quaternary history. Fournal of the Geological Society of London, 131, 19-37. 
HUNTER, J. R., 1975. A note on the quadratic friction in the presence of tides. Estuarine and Coastal Marine Science, 3, 473-475.

PINGREE, R. D. \& MADDOCK, L., 1977a. Tidal residuals in the English Channel. Fournal of the Marine Biological Association of the United Kingdom, 57, 339-354.

Pingree, R. D. \& MADDOCK, L., 1977 $b$. Tidal eddies and coastal discharge. Fournal of the Marine Biological Association of the United Kingdom, 57, 869-875.

SAUNDERS, P. M., 1977. Average drag in an oscillatory flow. Deep-Sea Research, 24, 381-384.

SHIELDS, A., 1936. Anwendung der Aehnlichkeitsmechanik und der Turbulenzforschung auf die Geschiebebewegung. Mitteilungen der Preubischen Versuchsanstalt für Wasserbau und Schiffbau, 26, 5-26. 